\title{
Research on the effect of resonant magnetic perturbations on disruption limit in
}

\section{J-TEXT tokamak}

Qiming $\mathrm{Hu}^{1,}$,, Nengchao Wang ${ }^{1,}$,, Q. Yu ${ }^{2}$, Yonghua Ding ${ }^{1}$, Bo Rao ${ }^{1}$, Zhipeng Chen $^{1}$, Hai Jin ${ }^{1}$

${ }^{1}$ State Key Laboratory of Advanced Electromagnetic Engineering and Technology, School of

Electric and Electronic Engineering, Huazhong University of Science and Technology, 430074, Wuhan, China

${ }^{2}$ Max-Planck-Institut für Plasmaphysik, 85748 Garching, Germany

${ }^{a}$ Corresponding author: huqm@hust.edu.cn and wangnc@ hust.edu.cn

Abstract. The effect of resonant magnetic perturbations (RMPs) on high density limit and low-q limit discharges is studied in J-TEXT tokamak. It is found that a moderate amplitude of applied RMPs increases the density limit and delays the disruption, and the disruption precursor is suppressed with a slight reduction in toroidal plasma rotation. A too large amplitude of RMPs, however, leads to a lower density limit and an earlier disruption. For the optimal RMPs amplitude, the disruption is delayed by about $150 \mathrm{~ms}$. For low- $q$ discharges, the applied static RMP can either lower the limit of the edge safety factor $q_{\mathrm{a}}$ from 2.15 to nearly 2.0 or sustain stable plasma discharge with $q_{\mathrm{a}}=2.1$, and the disruptive precursor is also suppressed.

\section{Introduction}

The feasibility and economics of a tokamak as a fusion reactor are strongly influenced by the occurrence of disruptions - rapid loss of all or a large fraction of the magnetic and thermal energy of the plasma column [1]. It is observed that many disruptions are preceded by the precursor MHD instability, a period of rotating magnetohydrodynamic (MHD) activity with low poloidal and toroidal mode numbers $[2,3]$. Near the disruption limit, the precursor MHD activity grows quickly and causes the disruption in several ms [1]. For example, the maximum current limit in a tokamak is set by the tearing-kink instability (usually $m=2, n=1$ ), which grows and disrupts the plasma when edge safety factor $q_{\mathrm{a}}$ approaches 2 [4], where $m$ and $n$ are the poloidal and toroidal mode numbers. While for given plasma current and minor radius, there is a maximum plasma density (namely density limit) that can be sustained [5], also set by a disruptive MHD-initiated instability.

Experiments had been performed in the past to control the precursor MHD activity and hence to avoid or delay the disruption by using resonant magnetic perturbations (RMPs). In 1970s, the disruptive instability was avoided by using the resonant-helical-field (RHF) in PULSATOR-I [6]. Later on, stabilization of disruption precursor had been extensively studied in other devices [7-9], and it was observed that RMPs can effectively reduce the width of magnetic islands [7, 8], and plasma stabilization had also been achieved by rotating RMPs [9]. In 1990s, RMPs feedback control had been used in the DITE tokamak for the stabilization of disruption precursors, and the disruptive density limit was significantly increased [10]. Recently, DIII-D and RFX-mod experiments have demonstrated stable tokamak operation at very low values of the edge safety factor $q_{\text {a }}$ near and 
below 2 via RMPs feedback control of MHD stability [11, 12]. In addition, it has been observed in TEXTOR that the threshold of the MARFE onset in the NBI heated plasmas is increased by making more homogeneous plasma recycling at the plasma edge by applying a $1 \mathrm{kHz}$ ac magnetic field perturbation at the high field side (HFS) [13]. These studies develop a flexible method to control disruption precursors.

Most of the precursor MHD instability before disruption is verified to be the tearing mode (TM) [1], hence it is important to control the precursor TM for the disruption mitigation/avoidance. Previous experimental studies showed that RMPs can suppress rotating TM effectively, accompanied by a plasma rotation reduction [14-17]. Theoretical analysis developed by Fitzpatrick $[18,19]$ reveals that the applied RMPs contribute a net stabilizing and braking effect on the TM and plasma rotation, respectively. The analytical results are confirmed by recent experimental and numerical studies in J-TEXT [16, 17, 20], which also indicate that RMPs can completely suppress the TM with partial rotation reduction. Further numerical modeling shows that a smaller amplitude TM can be suppressed more easily by RMPs [16], agreeing with Fitzpatrick's theory and suggesting that it is possible to take advantage of RMPs to control the precursor TM for disruption mitigation. In Fitzpatrick's analytical results the stabilizing and braking terms are [18]

$$
\begin{aligned}
& \Delta_{\mathrm{RMP}}^{\prime}=A_{1}\left(\frac{W_{v a c}}{W}\right)^{2} \cos \Phi, \\
& T_{z E M, \mathrm{RMP}}=A_{2} W_{v a c}^{2} W^{2} \sin \Phi,
\end{aligned}
$$

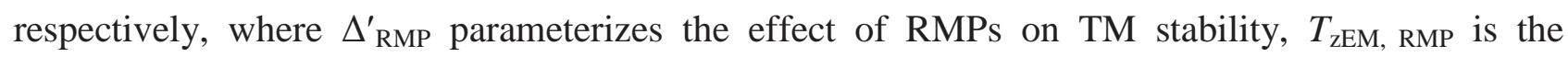
electromagnetic torque exerted by RMPs, $W_{\text {vac }}$ is the vacuum magnetic island width and represents the amplitude of RMPs, $W$ is the actual magnetic island width, $\Phi$ is the phase difference between magnetic island and RMPs, and $A_{1}$ and $A_{2}$ are constant parameters related to plasma equilibrium. The time integration of equations (1) and (2) reveals a net stabilizing and braking effect by the applied RMPs [19, 20]. The mechanism for the stabilization of TM by the RMP was described by Hender and Fitzpatrick $[14,18]$ : Due to the electromagnetic force applied from the RMP, the TM roation becomes non-uniform in a period, rotating faster in the half period when $\pi<\Phi<2 \pi$ but slower in another half period when $0<\Phi<\pi$ On the other hand, the RMP has a stabilizing (distabilizing) effect on the TM when $\Phi>|\pi / 2|(\Phi<|\pi / 2|)$. The non-uniform rotation leads to the TM (or magnetic island) to stay for a longer time in the half period when the RMP is stabilizing $(\Phi>|\pi / 2|)$, so that on the average the RMP has a net stabilizing effect on the mode. Such an effect is larger if the island width $W$ is smaller, as can be seen from Eq. (1). Therefore, in order to suppress the precursor TM with less rotation braking, for given amplitude of RMPs the island width $W$ should be as small as possible, so as to ensure a larger stabilizing effect while a weaker braking effect. For satisfying this requirement, it is better to apply the RMPs in the early growth phase of the precursor TM when the island width is small.

Motivated by the above understanding of the RMP effect on TM dynamics, experiments have 
been carried out in J-TEXT tokamak to study the RMPs effect on high density limit and low-q limit discharges, as reported in this paper, to control the disruptive precursors for disruption mitigation. In these experiments, the static RMPs are applied before the growth of precursor TM in both density limit and low- $q$ discharges. As a result, the density limit is increased and the low- $q$ limit is lowered, and the disruption is delayed, because the precursor TM is completely suppressed with a slight rotation reduction, as expected $[14,18]$ The experimental setup and results are presented in section 2, and the discussion and summary are given in section 3 .

\section{Experiment results}

\subsection{Experimental setup}

J-TEXT is a circular, medium sized, limiter tokamak with a major radius $\left(R_{0}\right)$ of $1.05 \mathrm{~m}$ and a minor radius $(a)$ of 0.25-0.3 m [21]. Recently, J-TEXT had been equipped with 12 in-vessel saddle coils located at four toroidal locations as shown in figure 1(a), which can generate either static or rotating RMPs [22]. In the present experiments, the coil system is set to only produce static RMPs, dominated by a resonant $m / n=2 / 1$ component with its radial magnetic field $b_{r}$ of the strength 2.5 Gs per kilo-ampere of coil current at plasma edge $(r=a)$. The electron density is measured with seven-channel far-infrared (FIR) HCN interferometer, which views the plasma vertically at $R=1.0$, $1.05,1.1,1.15,1.2,1.25$ and $1.3 \mathrm{~m} \mathrm{[23]}$ as shown in figure 1(b). The electron density provided by the channel at $R=1.05 \mathrm{~m}$ is used to interpret the experimental results in the context. The MHD activity is measured by the poloidal and toroidal Mirnov arrays as well as the locked mode detectors (figure 1(a)), which consist of two saddle coils installed at two opposite toroidal locations outside the vacuum vessel, and the difference between two saddle coil signals gives the perturbations with odd toroidal mode number $(n=1,3,5, \ldots)$. The edge toroidal rotation of $\mathrm{CV}$ impurity is measured by a multi-channel spectrometer [24].
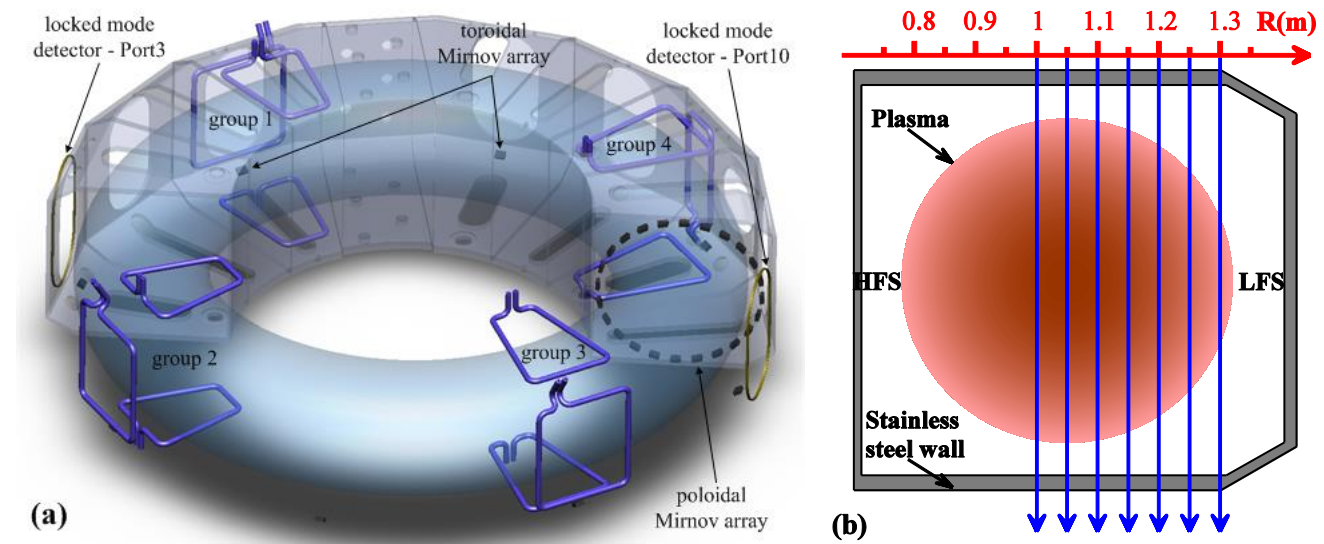

Figure 1. (a) Structure and layout of the DRMP coils, poloidal Mirnov array, toroidal Mirnov array, and locked mode detector on the J-TEXT tokamak [22]. (b) Diagram of seven-channel FIR HCN interferometer system for electron density measurement, which views the plasma vertically at $\mathrm{R}=1.0,1.05,1.1,1.15,1.2,1.25$ and $1.3 \mathrm{~m}$ on the low-field side (LFS).

For the experimental results presented below, two kinds of plasma conditions are adopted, and the corresponding plasma parameters are as follows unless otherwise noted: (I) density limit condition, major/minor radius $\left(R_{0} / a\right)$ is $1.05 \mathrm{~m} / 0.255 \mathrm{~m}$, plasma current $I_{\mathrm{p}}=140 \mathrm{kA}$, toroidal 
magnetic field $B_{\mathrm{t}}=1.6 \mathrm{~T}$, the safety factor $q_{\mathrm{a}}=3.55$ at the plasma edge, the central chord line-averaged electron density at $R=1.05 \mathrm{~m}$ is ramped up in the range $3-6 \times 10^{19} \mathrm{~m}^{-3}$ until disruption, and the Greenwald density limit is $n_{\mathrm{G}}=I_{\mathrm{p}} / \pi a^{2}=6.85 \times 10^{19} \mathrm{~m}^{-3}$. (II) low- $q$ limit condition, major/minor radius $\left(R_{0} / a\right)$ is $1.05 \mathrm{~m} / 0.255 \mathrm{~m}$, the central chord line-averaged electron density at $R=1.05 \mathrm{~m}$ is around $1.0 \times 10^{19} \mathrm{~m}^{-3}$, the toroidal magnetic field $B_{\mathrm{t}}=1.35-1.55 \mathrm{~T}$, the plasma current is ramped up from $180 \mathrm{kA}$ to $215 \mathrm{kA}$ after reaching its flattop, and the edge safety factor is lowered from $q_{\mathrm{a}}=2.65$ to 1.95 . For all cases reported in this paper, an $m / n=2 / 1$ TM grows quickly when approaching the high density or low-q limit, and it rotates in the counter- $I_{\mathrm{p}}$ direction with a frequency decreasing from $10 \mathrm{kHz}$ to $2 \mathrm{kHz}$. The central plasma rotation is observed to be in the electron diamagnetic drift direction.

\subsection{The effect of RMPs on density limit}

A typical example of the density limit discharge is shown in figure 2 for shot 1033264 without the application of RMPs. The effective charge $Z_{\text {eff }}$ is about 2.5 (figure 2(b)) measured by the spectrometer system. In the experiments, electron density is kept increasing by gas puffing. During the interval of $t<0.32 \mathrm{~s}$, the line-averaged electron density $n_{\mathrm{e}}$ keeps increasing to $4.6 \times 10^{19} \mathrm{~m}^{-3}$ (figure 2(a)), and there is a magnetic fluctuation with a frequency about $10 \mathrm{kHz}$ (figure 2(b) and 1(d)). This magnetic fluctuation is found to be an $m / n=2 / 1 \mathrm{TM}$ [25]. The toroidal rotation of CV impurity is $\mathrm{V}_{\mathrm{CV}}=-10 \mathrm{~km} / \mathrm{s}$, here $\mathrm{V}_{\mathrm{CV}}$ is the toroidal rotation at the radial location $r=0.75 \sim 0.85 a$, and the negative value of $\mathrm{V}_{\mathrm{CV}}$ indicates the rotation in the counter- $I_{\mathrm{p}}$ direction. During the interval of $0.32 \mathrm{~s}<t<0.33 \mathrm{~s}, n_{\mathrm{e}}$ is further increased to $4.7 \times 10^{19} \mathrm{~m}^{-3}=0.68 n_{\mathrm{G}}$, and the magnetic fluctuation grows to an amplitude of more than $15 \mathrm{Gs}$ as shown by the magnetic perturbation $\delta B_{\theta}{ }^{\mathrm{n}=1}$ in figure 2(b). Meanwhile the frequency of the precursor TM decreases from $10 \mathrm{kHz}$ to $1 \mathrm{kHz}$, and mode locking and disruption happen shortly afterwards as shown by figure $2(\mathrm{~d})$. And $\mathrm{V}_{\mathrm{CV}}$ changes from the initial value of $-10 \mathrm{~km} / \mathrm{s}$ to a positive value in the co- $I_{\mathrm{p}}$ direction (figure $2(\mathrm{c})$ ), which is similar to previous observation in mode penetration experiments in COMPASS-C [14]. In J-TEXT, the precursor TM is usually observed before density limit disruption, and the density limit is found to be less than $0.7 n_{\mathrm{G}}$. 


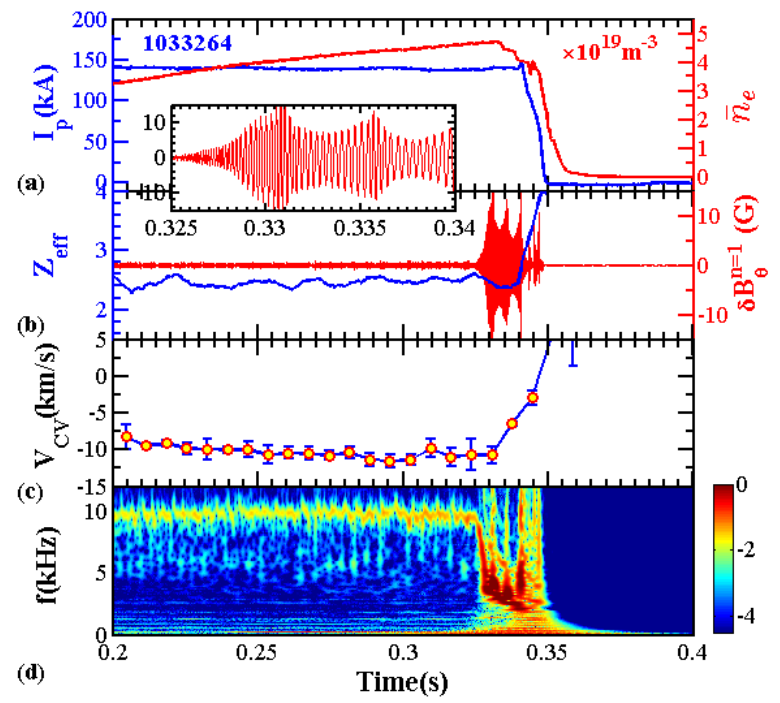

Figure 2. Typical density limit disruption in J-TEXT. Time traces of (a) plasma current $I_{\mathrm{p}}$ (blue curve) and line-averaged electron density $\bar{n}_{e}$ (red curve), (b) effective $Z_{\text {eff }}$ (blue curve) and $n=1$ magnetic perturbation $\delta B_{\theta}{ }^{\mathrm{n}=1}$ (red curve), (c) toroidal rotation of $\mathrm{CV}$ impurity $\mathrm{V}_{\mathrm{CV}}$ and (d) wavelet power spectrum of Mirnov signal.
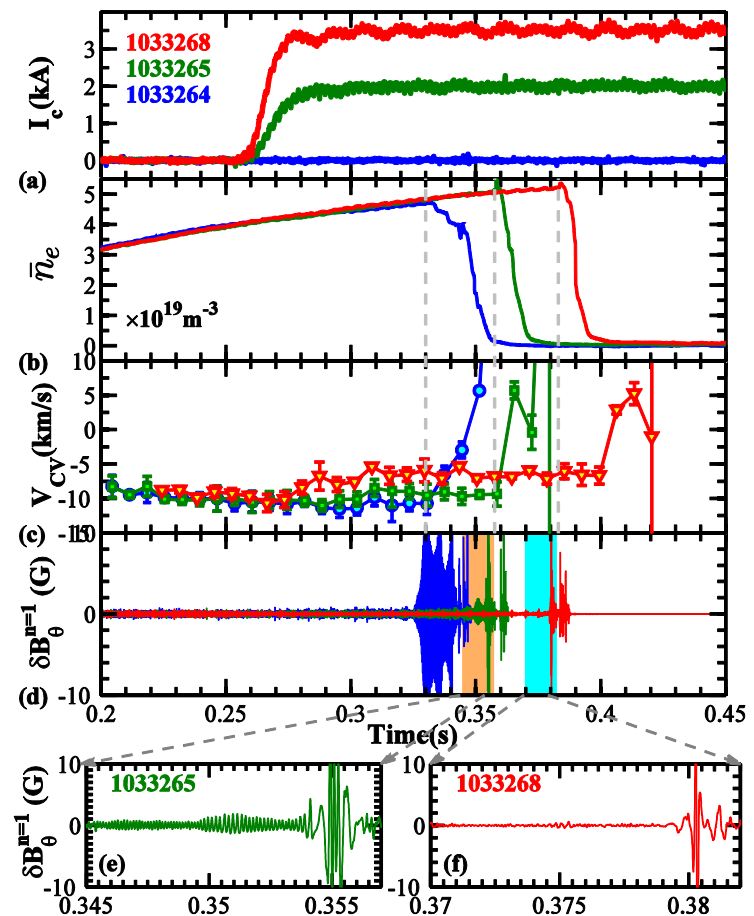

Figure 3. Influence of static RMP on density limit. Time traces of (a) static RMPs current $I_{\mathrm{c}}$, (b) line-averaged electron density $\bar{n}_{e}$, (c) plasma toroidal rotation of $\mathrm{CV}$ impurity $\mathrm{V}_{\mathrm{CV}}$, (d) $n=1$ magnetic perturbation $\delta B_{\theta}{ }^{\mathrm{n}=1}$ for shots 1033264 , 1033265 and 1033268, and detail evolution of $\delta B_{\theta}{ }^{\mathrm{n}=1}$ for shot (e) 1033265 and (f) 1033268.

In the following experiments, the static RMPs are applied before the onset of the precursor TM as shown in figure 3. The case without RMPs (shot 1033264) is also shown there for comparison. The static RMPs coil current $\left(I_{\mathrm{c}}\right)$ is turned on at $t=0.26 \mathrm{~s}$, then ramping up to $2 \mathrm{kA}$ in $0.02 \mathrm{~s}$ for shot 1033265 and to $3.5 \mathrm{kA}$ in $0.02 \mathrm{~s}$ for shot 1033268 (figure 3(a)), respectively. During the application of a weaker static RMPs in shot 1033265, the core line-averaged electron density increases to $n_{\mathrm{e}}=$ $5.05 \times 10^{19} \mathrm{~m}^{-3}=0.74 n_{\mathrm{G}}$, and then disruption happens at $t=0.36 \mathrm{~s}$. When looking into the time evolution of $\delta B_{\theta}{ }^{\mathrm{n}=1}$ in figure 3(d) and 3(e), it is found that $\delta B_{\theta}{ }^{\mathrm{n}=1}$ is less than 2 Gs before disruption. In addition, the toroidal rotation $\mathrm{V}_{\mathrm{CV}}$ decreases slightly from $-10.5 \mathrm{~km} / \mathrm{s}$ to $-9.4 \mathrm{~km} / \mathrm{s}$ before disruption. Compared to the case without RMPs (shot 1033264), for $I_{\mathrm{c}}=2 \mathrm{kA}$ the limit density is increased by $7.5 \%$ with the time of disruption being delayed by $30 \mathrm{~ms}$. The amplitude of precursor TM is substantially suppressed, and the plasma rotation is reduced little. When a larger amplitude of static RMPs are applied for shot 1033268 with $I_{\mathrm{c}}=3.5 \mathrm{kA}$ at the flattop, the core density is further increased to $n_{\mathrm{e}}=5.25 \times 10^{19} \mathrm{~m}^{-3}=0.77 n_{\mathrm{G}}$, and the disruption occurs at $t=0.38 \mathrm{~s}$. The time evolution of $\delta B_{\theta}{ }^{\mathrm{n}=1}$ in figure 3(d) and 3(f) show that there is no precursor TM until disruption, indicating that it is completely suppressed. Besides, toroidal rotation $\mathrm{V}_{\mathrm{CV}}$ decreases from $-10.1 \mathrm{~km} / \mathrm{s}$ to $-7.2 \mathrm{~km} / \mathrm{s}$. Comparing to the case without RMPs, the limit density is increased by $12 \%$ with the time of disruption being delayed by $50 \mathrm{~ms}$. For both cases with RMPs, the disruptions are eventually caused by field penetration. 


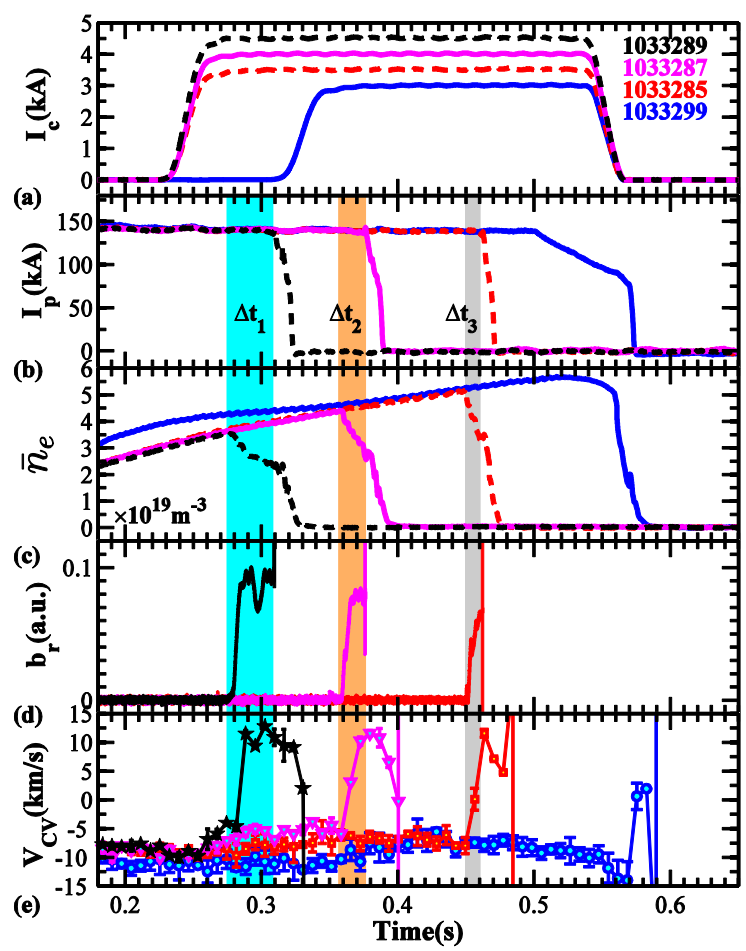

Figure 4. Effect of RMP amplitude on density limit. Time traces of (a) static RMPs current $I_{\mathrm{c}}$, (b) plasma current $I_{\mathrm{p}}$, (c) line-averaged electron density $\bar{n}_{e}$, (d) the locked mode detector signals $b_{\mathrm{r}}$ and (e) plasma toroidal rotation of CV impurity $\mathrm{V}_{\mathrm{CV}}$ for shots 1033299 (blue), 1033285 (red), 1033287 (pink) and 1033289 (black).

The results shown in figure 3 verify that the static RMPs effectively suppress the precursor TM, increase the limit density and delay the occurrence of disruption for tens of $m s$. RMPs with a too strong amplitude, however, decreases the limit density and leads to earlier occurrence of disruption, as shown in figure 4. The static RMPs coil current $\left(I_{\mathrm{c}}\right)$ is $3 \mathrm{kA}$ for shot $1033299,3.5 \mathrm{kA}$ for shot 1033285, $4 \mathrm{kA}$ for shot 1033287, and $4.5 \mathrm{kA}$ for shot 1033289. The disruption occurs during the ramp down phase of plasma current for shot 1033299. The density is $n_{\mathrm{e}}=5.1 \times 10^{19} \mathrm{~m}^{-3}$ when the plasma current is $90 \mathrm{kA}\left(n_{\mathrm{G}}=4.4 \times 10^{19} \mathrm{~m}^{-3}\right)$, transiently exceeding the Greenwald limit. For shots 1033285, 1033287 and 1033289 shown in figure 4, field penetration occurs as indicated by the rapid growth of locked mode detector signal $b_{\mathrm{r}}$ (figure $4(\mathrm{~d})$ ). As a result the large magnetic island first causes the density to decrease [26], and then the current quench occurs 10-30 ms later. The time interval between these two time points are shadowed and marked by $\Delta t_{1}, \Delta t_{2}$ and $\Delta t_{3}$ respectively $\left(\Delta t_{1}=35 \mathrm{~ms}\right)$. The plasma rotation is slightly decreased during the suppression phase of precursor TM with a rotation reduction $\Delta \mathrm{V}_{\mathrm{CV}} \sim 3 \mathrm{~km} / \mathrm{s}$, and plasma rotation reversion to the co- $I_{\mathrm{p}}$ direction after field penetration [14]. The results of figure 4 are summarized in table 1. RMPs results in lower limit density when $I_{\mathrm{c}}>3.5 \mathrm{kA}$. In table 1 the density for shot 1033299 is the maximum value during the flattop of plasma current but not the one during the ramp down phase of plasma current. The wavelet power spectra of the Mirnov signal for these four shots are shown in figure 5, which shows that the precursor TM is partially suppressed for shot 1033299, completely suppressed for shots 1033285,1033287 and 1033289. 
Table 1 . Summary of the parameters for the 4 shots corresponding to figure 4 .

\begin{tabular}{|c|c|c|c|c|c|c|c|}
\hline \multirow{2}{*}{ shot $\mathrm{No}$} & \multirow[b]{2}{*}{$\mathbf{I}_{\mathbf{c}}(\mathbf{k A})$} & \multirow[b]{2}{*}{$n_{\mathrm{e}}\left(\times 10^{19} \mathrm{~m}^{-3}\right)$} & \multirow[b]{2}{*}{$n_{\mathrm{e}} / n_{\mathrm{G}}$} & \multirow{2}{*}{$\begin{array}{c}\text { Disruption } \\
\text { time (s) }\end{array}$} & \multicolumn{3}{|c|}{$\mathrm{V}_{\mathrm{CV}}(\mathrm{km} / \mathrm{s})$} \\
\hline & & & & & Initial & $\begin{array}{c}\text { During } \\
\text { suppression } \\
\end{array}$ & $\begin{array}{c}\text { After } \\
\text { penetration }\end{array}$ \\
\hline 1033299 & 3 & 5.7 & 0.83 & 0.57 & -11.3 & -8.1 & 2 \\
\hline 1033285 & 3.5 & 5.15 & 0.75 & 0.45 & -9.5 & -6.8 & 11.4 \\
\hline 1033287 & 4 & 4.4 & 0.64 & 0.36 & -9.6 & -5.9 & 11.5 \\
\hline 1033289 & 4.5 & 3.5 & 0.51 & 0.28 & -9.8 & -4.6 & 12.7 \\
\hline
\end{tabular}
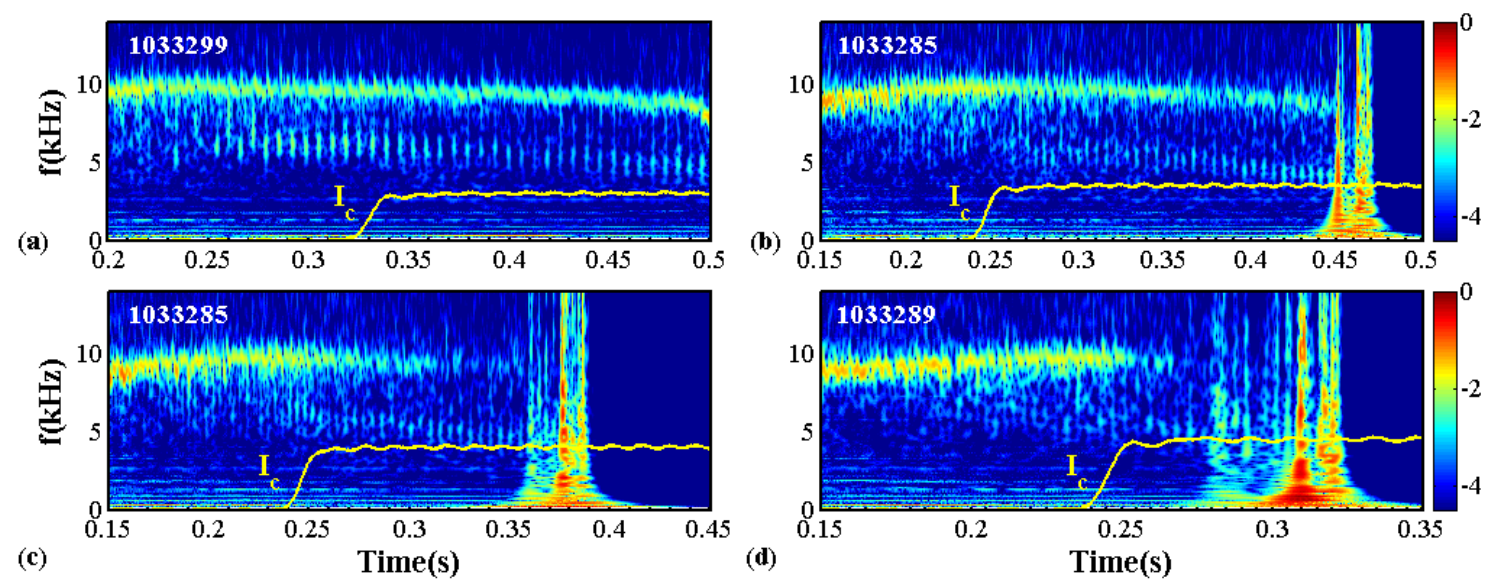

Figure 5. Corresponding to figure 4, wavelet power spectrum of Mirnov signal for shots (a) 1033299, (b) 1033285, (c) 1033287 and (d) 1033289 are shown together with RMPs current $I_{\mathrm{c}}$.

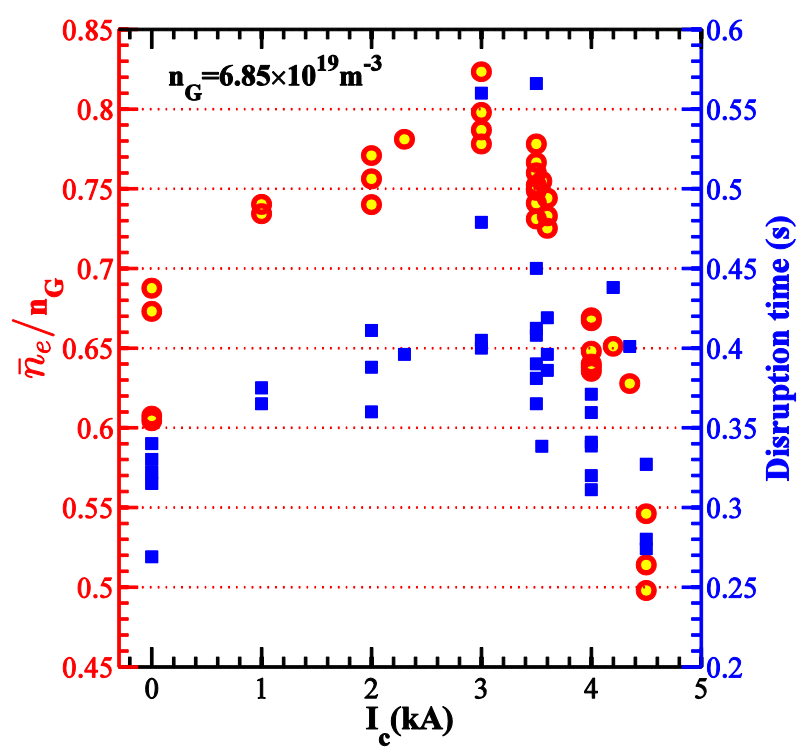

Figure 6. Ratio between $n_{\mathrm{e}}$ and Greenwald density $n_{\mathrm{G}}$ (red circles) and the disruption time (blue squares) versus static RMPs current $I_{\mathrm{c}}$.

Systematical experiments have been carried out by keeping the background plasma parameters to be the same, while only the amplitude of static RMPs are changed by scanning $I_{\mathrm{c}}$ from $1 \mathrm{kA}$ to $4.5 \mathrm{kA}$. In figure $6, n_{\mathrm{e}} / n_{\mathrm{G}}$ and the disruption time are shown as a function of $I_{\mathrm{c}}$. The density is usually limited to $n_{\mathrm{e}}<0.68 n_{\mathrm{G}}$ and disruption happens at $t<0.33 \mathrm{~s}$ without the application of static RMPs. Both the limit density and disruption time linearly increase with $I_{\mathrm{c}}$ for $I_{\mathrm{c}} \leq 3 \mathrm{kA}$. Static RMPs increase the limit density to more than $0.8 n_{\mathrm{G}}$, and the disruption is delayed by $30 \mathrm{~ms}$ to 100 $\mathrm{ms}$ for $I_{\mathrm{c}}=3 \mathrm{kA}$. However, even stronger RMPs do not lead to a further increase in the limit density 
but a decrease in it with an earlier disruption. The limit density decreases from about $\sim 0.8 n_{\mathrm{G}}$ to $0.5 n_{\mathrm{G}}$ when $I_{\mathrm{c}}$ increases from $3 \mathrm{kA}$ to $4.5 \mathrm{kA}$. It should be noted that the intrinsic $2 / 1$ error field in J-TEXT is measured to be $0.3 \mathrm{Gs}$ at the plasma edge $r=a$ [27], being much weaker than applied static RMPs (ranging from 2.5 Gs to $11 \mathrm{Gs}$ ), and the toroidal phases of intrinsic error field $\left(317^{\circ}\right.$ ) and static RMPs $\left(290^{\circ}\right)$ are close. Therefore, the increased limit density is not due to the correction of error field. The maximum density is obtained due to the presence of static RMPs with an appropriate amplitude $\left(I_{\mathrm{c}}=3 \mathrm{kA}\right)$.

\subsection{The effect of RMPs on low-q limit}

Similar to the results presented in section 2.2, static RMPs are also applied in the low- $q$ discharges. In order to approach the low- $q$ limit, the plasma current is ramped up slowly for $100 \mathrm{~ms}$ after reaching its first flattop at $I_{\mathrm{p}}=180 \mathrm{kA}$. The experimental results without RMPs are shown in figure 7 with the toroidal magnetic field $B_{\mathrm{t}}=1.55 \mathrm{~T}$ and $1.35 \mathrm{~T}$ for shots 1037639 and 1037647 , respectively. It is seen that disruption does not happen for shot 1037639 with minimum $q_{\mathrm{a}}=2.2$, and there is no obvious precursor TM in $\delta B_{\theta}{ }^{\mathrm{n}=1}$. While for shot 1037647, disruption happens when $q_{\mathrm{a}}$ approaches 2.15. The time evolution of $\delta B_{\theta}{ }^{\mathrm{n}=1}$ reveals that an $m / n=2 / 1$ precursor TM is excited just before the disruption, and the mode frequency is around $10 \mathrm{kHz}$, being similar to that of density limit discharges. In J-TEXT, the precursor $2 / 1 \mathrm{TM}$ is often observed in low- $q$ limit disruption, and the limit $q_{\mathrm{a}}$ is found to be around 2.15-2.2.

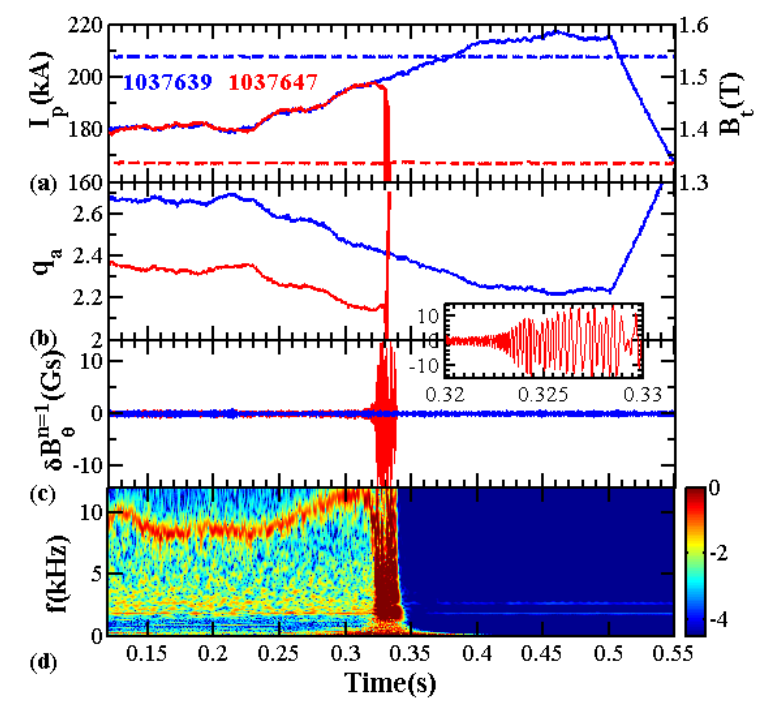

Figure 7. Typical low- $q$ discharges in J-TEXT. Time traces of (a) plasma current $I_{\mathrm{p}}$ (solid curves) and toroidal field $B_{\mathrm{t}}$ (dashed curves), (b) edge safety factor $q_{\mathrm{a}}$, (c) $n=1$ magnetic perturbation $\delta B_{\theta}{ }^{\mathrm{n}=1}$ for shots 1037639 (blue) and 1037647 (red), and (d) wavelet power spectrum of Mirnov signal for shot 1037647.

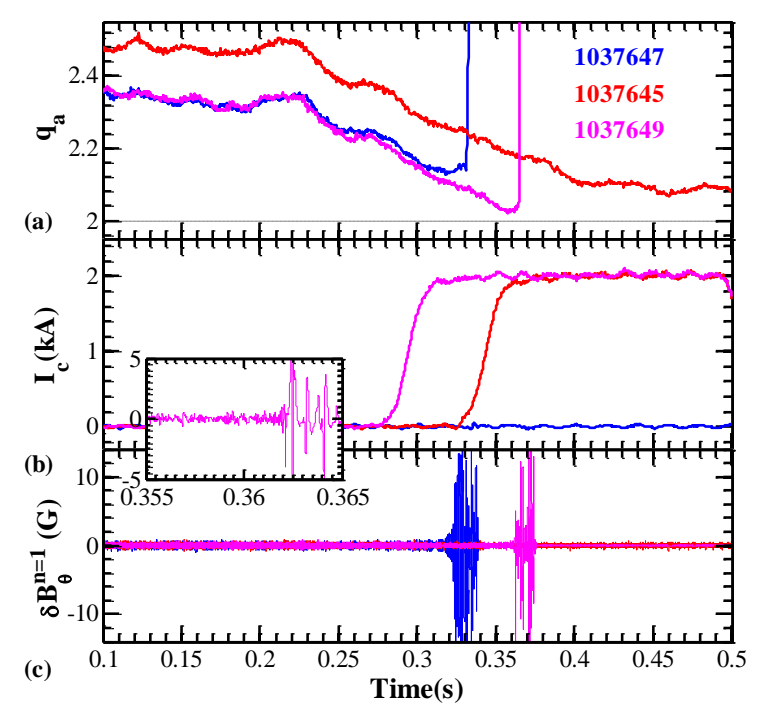

Figure 8. Influence of static RMP on low- $q$ discharge. Time traces of (a) edge safety factor $q_{\mathrm{a}}$, (b) static RMP current $I_{\mathrm{c}}$ and (c) $n=1$ magnetic perturbation $\delta B_{\theta}{ }^{\mathrm{n}=1}$ for shots 1037647,1037645 and 1037649.

Figure 8 shows the effect of static RMPs on low- $q$ discharges for shots 1037645 and 1037649. The static RMPs is applied when $q_{\mathrm{a}}$ decreases to 2.2 with $I_{\mathrm{c}}=2 \mathrm{kA}$ at the flattop. Compared to the case without RMPs (shot 1037647), disruption does not occur for shot 1037645 with a lower $q_{\mathrm{a}}, q_{\mathrm{a}}$ $=2.1$. For shot 1037649, disruption happens until $q_{\mathrm{a}}$ approaching 2.0. The evolution of $\delta B_{\theta}{ }^{\mathrm{n}=1}$ just 
before disruption shown in figure 8(c) reveals that the precursor TM is also completely suppressed. The results shown in figure 8 reveal that static RMPs can lower the low- $q$ limit from 2.15 to 2 and suppress the precursor TM. Additional studies have also been carried out to look into the effect of RMPs amplitude, it is found that lowest $q_{\mathrm{a}}, q_{\mathrm{a}}=2$ is approached for $1 \mathrm{kA} \leq I_{\mathrm{c}} \leq 2.5 \mathrm{kA}$, while stronger RMPs trigger the disruption at higher $q_{\mathrm{a}}$. However, the $q_{\mathrm{a}}=2$ limit has never been overcome. The required amplitude of RMPs to complete suppress precursor TM for low- $q$ discharges is smaller than that of density limit discharges, since the $q=2$ is closer to plasma edge in this case.

\section{Discussion and summary}

The effect of resonant magnetic perturbations (RMPs) on high density limit and low-q limit discharges is studied in J-TEXT tokamak. It is found that a moderate amplitude of applied RMPs increase the density limit from less than $0.7 n_{\mathrm{G}}$ to more than $0.8 n_{\mathrm{G}}$ for $I_{\mathrm{c}} \leq 3 \mathrm{kA}$, and the disruption is delayed by about $30 \mathrm{~ms}$ to $100 \mathrm{~ms}$. The disruptive precursor is suppressed with a little change in toroidal rotation. A too large amplitude of RMPs with $I_{\mathrm{c}}>3.5 \mathrm{kA}$, however, leads to a lower density limit and an earlier occurrence of disruption. For the optimal RMPs amplitude with $I_{\mathrm{c}}=3 \mathrm{kA}, n_{\mathrm{e}}=$ $0.83 n_{\mathrm{G}}$ is achieved, and the disruption is delayed by about $150 \mathrm{~ms}$. The toroidal plasma rotation of $\mathrm{CV}$ impurity decreases a little during the suppression phase of precursor TM with $\Delta \mathrm{V}_{\mathrm{CV}} \sim 3 \mathrm{~km} / \mathrm{s}$. For low- $q$ discharges, the applied static RMP can either lower the edge safety factor $q_{\mathrm{a}}$ from 2.15 to nearly 2.0 or sustain stable plasma discharge with $q_{\mathrm{a}}=2.1$, and the disruptive precursor is also suppressed.

These experimental results are consistent with the mechanism of TM stabilization by static RMPs described in section 1. In the high density limit and low-q limit discharges on J-TEXT tokmark, the disruptions are caused by the growth of the TM and subsequent mode locking, similar to the observations from other tokmarks. A moderate amplitude of RMP delays the disruption because it has a stabilizing effect on the rotating TM, as seen from the experimental results. A too large amplitude of the RMP, however, will lead to field penetration (locked mode) and therefore an earlier disruption, even if the precursor TM is stabilized.

The studies presented in this paper reveal several features and possible applications of externally applied RMPs. Firstly, the optimal amplitude of RMPs to obtain the maximum density or minimum $q_{\mathrm{a}}$ is not zero, indicating that a finite amplitude of error field (or RMPs) is in fact stabilizing in these cases. Secondly, together with the disruption prediction system, static RMPs can be utilized for delaying the disruption, to allow more time ( $\sim$ tens $-100 \mathrm{~ms}$ ) for disruption mitigation by using, e.g., massive gas injection. Thirdly, as static RMPs can effectively suppress the rapid growth of TM instability, it might also be able to suppress the growth of NTM (neoclassical TM) [28] seed island if RMPs is applied in its early growing phase. Fourthly, the amplitude of RMPs to excite field penetration in density limit discharge is lower than the error field penetration threshold observed in J-TEXT before [25], indicating that the field penetration threshold depends on other plasma parameters in addition to the plasma density. 
RMPs has been used to suppress the runaway electron beam on TEXTOR [29, 30], and it is also proposed for runaway mitigation on ITER $[31,32]$. It is possible to both delay the disruption and suppress the runaway electron by applying an appropriate waveform of RMPs.

It should be noted that the static RMPs are applied much earlier before the growth of precursor TM in our experiments, because the magnetic feedback has not been utilized in the presented experiments. In addition, during the application of static RMPs, a discharge with higher and stable electron density has not been tried except for shot 1033299, and this will be carried out in the future experiment.

In summary, static RMPs are used to influence the disruption limit and control disruption precursors in both density limit and low- $q$ limit discharges in J-TEXT Ohmic plasmas. The major results are as follows:

(1) A moderate amplitude of applied RMPs increases the density limit and delays the disruption. The disruption precursor is suppressed with a slight reduction in toroidal plasma rotation. A too large amplitude of RMPs, however, leads to a lower density limit and an earlier disruption. For the optimal RMPs amplitude, the disruption is delayed by about $150 \mathrm{~ms}$.

(2) By applying a moderate amplitude of RMPs, the edge safety factor $q_{\mathrm{a}}$ is lowered from 2.15 to 2.0 .

(3) Precursor 2/1 TM is effectively suppressed for both density limit and low-q limit discharges.

(4) The toroidal plasma rotation $\mathrm{V}_{\mathrm{CV}}$ is slightly decreased in the suppression phase of precursor $\mathrm{TM}$ with $\Delta \mathrm{V}_{\mathrm{CV}} \sim 3 \mathrm{~km} / \mathrm{s}$.

\section{Acknowledgement:}

The authors would like to thank the members of the J-TEXT team for their assistance in the experiment. This work is supported by National Magnetic Confinement Fusion Science Program of China (Contract No. 2015GB111001, 2014GB118000 and 2013GB106003) and the National Natural Science Foundation of China (Contract No.11275080 and 11505069).

\section{References}

[1] Wesson J. A. et al 1989 Nuclear Fusion 29641

[2] Basis I. P. 1999 Nuclear Fusion 392251

[3] de Vries P. C. et al 2011 Nuclear Fusion 51053018

[4] Wesson J. A. 1978 Nuclear Fusion 1887

[5] Martin G. 2002 Plasma Physics and Controlled Fusion 44 R27

[6] Karger F. et al 1975 Influence of resonant helical fields on tokamak discharges. In: Proceeding of the 5th IAEA Int. Conf. on Plasma Physics and Controlled Nuclear Fusion Energy Research (Tokyo, Japan,1974), p 207

[7] Ellis J. J. et al 1985 The influence of resonant helica fields on tokamak confinement. In: Proc. 10th IAEA Int. Conf. on Plasma Physics and Controlled Nuclear Fusion Energy Research (London, UK, 1984), p 363

[8] Chen J. et al 1990 Nuclear Fusion 302271

[9] Arsenin V. V. et al 1979 Feedback stabilization of kink instability in the TO-1 tokamak. In: Proc. 7th IAEA Int. Conf. on Plasma Physics and Controlled Nuclear Fusion Energy Research (Innsbruck, Austria, 1978), p 233

[10] Morris A. W. et al 1990 Physical Review Letters 641254 
[11] Piovesan P. et al 2014 Physical Review Letters 113045003

[12] Hanson J. M. et al 2014 Physics of Plasmas 21

[13] Liang Y. et al 2005 Physical Review Letters 94105003

[14] Hender T. C. et al 1992 Nuclear Fusion 322091

[15]Elgriw S. et al 2011 Nuclear Fusion 51113008

[16] Hu Q. et al 2012 Nuclear Fusion 52083011

[17] Rao B. et al 2013 Physics Letters A 377315

[18] Fitzpatrick R. 1993 Nuclear Fusion 331049

[19] Fitzpatrick R. et al 2001 Physics of Plasmas 84489

[20] Hu Q. et al 2013 Physics of Plasmas 20092502

[21]Zhuang G. et al 2011 Nuclear Fusion 51094020

[22] Rao B. et al 2014 Fusion Engineering and Design 89378

[23] Gao L. et al 2012 Review of Scientific Instruments 83 10E303

[24] Cheng Z. F. et al 2013 Review of Scientific Instruments 84073508

[25] Wang N. et al 2014 Nuclear Fusion 54064014

[26] Hu Q. et al 2014 Nuclear Fusion 54064013

[27] Rao B. et al 2013 Review of Scientific Instruments 84043504

[28] Fietz S. et al 2015 Nuclear Fusion 55013018

[29] Finken K H. et al 2006 Nuclear Fusion 46 S139-44

[30] Lehnen M. et al 2008 Physical Review Letters 100255003

[31] Papp G. et al 2011 Plasma Physics and Controlled Fusion 53095004

[32] Papp G. et al 2012 Plasma Physics and Controlled Fusion 54125008 